\title{
Limited Codes Associated with Petri Nets
}

\author{
Genjiro Tanaka*
}

\begin{abstract}
The purpose of this paper is to investigate the relationship between limited codes and Petri nets. The set $M$ of all positive firing sequences which start from the positive initial marking $\mu$ of a Petri net and reach $\mu$ itself forms a pure monoid $M$ whose base is a bifix code. Especially, the set of all elements in $M$ which pass through only positive markings forms a submonoid $N$ of $M$. Also $N$ has a remarkable property that $N$ is pure. Our main interest is in the base $D$ of $N$. The family of pure monoids contains the family of very pure monoids, and the base of a very pure monoid is a circular code. Therefore, we can expect that $D$ may be a limited code. In this paper, we examine "small" Petri nets and discuss under what conditions $D$ is limited.
\end{abstract}

Keywords: free monoid, Petri net, code, prefix code, circular code, limited code

\section{Introduction}

Let $A$ be an alphabet, $A^{*}$ the free monoid over $A$, and 1 the empty word. Let $A^{+}=A^{*}-\{1\}$. A word $v \in A^{*}$ is a right factor of a word $u \in A^{*}$ if there is a word $w \in A^{*}$ such that $u=w v$. The right factor $v$ of $u$ is called proper if $v \neq u$. For a word $w \in A^{*}$ and a letter $x \in A$ we let $|w|_{x}$ denote the number of $x$ in $w$. The length $|w|$ of $w$ is the number of letters in $w$.

A non-empty subset $C$ of $A^{+}$is said to be a code if for $x_{1}, \ldots, x_{p}, y_{1}, \ldots, y_{q} \in$ $C, p, q \geq 1$,

$$
x_{1} \cdots x_{p}=y_{1} \cdots y_{q} \Longrightarrow p=q, x_{1}=y_{1}, \ldots, x_{p}=y_{p} .
$$

A subset $M$ of $A^{*}$ is a submonoid of $A^{*}$ if $M^{2} \subseteq M$ and $1 \in M$. Every submonoid $M$ of a free monoid has a unique minimal set of generators $C=(M-\{1\})-(M-\{1\})^{2}$. $C$ is called the base of $M$. A submonoid $M$ is right unitary in $A^{*}$ if for all $u, v \in A^{*}$,

$$
u, u v \in M \Longrightarrow v \in M \text {. }
$$

${ }^{*}$ Dept. of Computer Science, Shizuoka Institute of Science and Technology, Fukuroi-shi, 4378555 Japan. E-mail: tanaka@cs.sist.ac.jp 
$M$ is called left unitary in $A^{*}$ if it satisfies the dual condition. A submonoid $M$ is biunitary if it is both left and right unitary.

Definition 1.1. Let $M$ be a submonoid of a free monoid $A^{*}$, and $C$ its base. If $C A^{+} \cap C=\emptyset$, (resp. $A^{+} C \cap C=\emptyset$ ), then $C$ is called a prefix (resp. suffix) code over $A$. $C$ is called a bifix code if it is a prefix and suffix code.

A submonoid $M$ of $A^{*}$ is right unitary (resp. biunitary) if and only if its minimal set of generator is a prefix code (resp. bifix code) $([1, \mathrm{p} .46],[3, \mathrm{p} .108])$.

Definition 1.2. A Petri net is a 4-tuple, $P N=\left(P, A, W, \mu_{0}\right)$ where $P=$ $\left\{p_{1}, p_{2}, \ldots, p_{m}\right\}$ is a finite set of places, $A=\left\{t_{1}, t_{2}, \ldots, t_{n}\right\}$ is a finite set of transitions such that $P \cap A=\emptyset$ and $P \cup A \neq \emptyset, W:(P \times A) \cup(A \times P) \rightarrow\{1,2, \ldots\}$ is a weight function, $\mu_{0}: P \rightarrow\{0,1,2, \ldots\}$ is the initial marking.

Let $t \in A$, and let $\cdot t=\{p \in P \mid(p, t) \in P \times A\}$ and $t \cdot=\{p \in P \mid(t, p) \in A \times P\}$. In this paper we shall assume that a Petri net has no isolated transitions, i.e., no $t$ such that $\cdot t \cup t \cdot=\emptyset$. A transition $t$ is said to be enabled in a marking $\mu_{0}$, if $W(p, t) \leq \mu_{0}(p)$ for all $p \in \cdot t$. A firing of an enabled transition $t$ removes $W(p, t)$ tokens from each input place $p \in \cdot t$, and adds $W(t, p)$ tokens to each output place $p \in t$. A firing of an enabled transition $t$ in $\mu_{0}$ produces a new marking $\mu_{1}$

$$
\mu_{1}(p)=\mu_{0}(p)-W(p, t)+W(t, p)
$$

for any $p \in P$, denoted by $\mu_{1}=\delta(\mu, t)$. A string $w=t_{1} t_{2} \ldots t_{r}, t_{i} \in A$, of transitions is said to be a (firing) sequence from $\mu_{0}$ if there exist markings $\mu_{i}, 1 \leq i \leq r$, such that $\delta\left(\mu_{i-1}, t_{i}\right)=\mu_{i}$ for all $i, 1 \leq i \leq r$. In this case, $\mu_{r}$ is reachable from $\mu_{0}$ by $w$ and we write $\delta\left(\mu_{0}, w\right)=\mu_{r}$. The set of all possible markings reachable from $\mu_{0}$ is denoted by $\operatorname{Re}\left(\mu_{0}\right)$, and the set of all possible sequences from $\mu_{0}$ is denoted by $\operatorname{Seq}\left(\mu_{0}\right)$. The function $\delta: \operatorname{Re}\left(\mu_{0}\right) \times A \rightarrow \operatorname{Re}\left(\mu_{0}\right)$ is called a next-state function of a Petri net $P N$ [5.p.23]. We note that the above condition for $r=0$ is understood to be $\mu_{0} \in R e\left(\mu_{0}\right)$. A marking $\mu$ is said to be positive if $\mu(p)>0$ for all $p \in P$. A sequence $t_{1} t_{2} \ldots t_{n} \in S e q\left(\mu_{0}\right), t_{i} \in A$, is called a positive sequence from $\mu_{0}$ if $\delta\left(\mu_{0}, t_{1} t_{2} \ldots t_{i}\right)$ is positive for all $i, 1 \leq i \leq n$. The set of all positive sequences from $\mu_{0}$ is denoted by $P \operatorname{Seq}\left(\mu_{0}\right)$.

Let $P=\left\{p_{1}, p_{2}, \cdots, p_{n}\right\}$. A marking $\mu$ can be represented by a vector $\mu=$ $\left(\mu\left(p_{1}\right), \mu\left(p_{2}\right), \cdots, \mu\left(p_{n}\right)\right)$. For every $t \in A$ the vector $\Delta t$ is defined by

$$
\Delta t=\left(\Delta t\left(p_{1}\right), \Delta t\left(p_{2}\right), \ldots, \Delta t\left(p_{n}\right)\right), n=|P|,
$$

where $\Delta t(p)=-W(p, t)+W(t, p)$. For a sequence $w=t_{1} t_{2} \ldots t_{n} \in \operatorname{Seq}\left(\mu_{0}\right)$ and $p \in P, \Delta w=\sum_{i=1}^{n} \Delta t_{i}$ and $\Delta w(p)$ is a $p$-th component of a vector $\Delta w$, i.e., $\Delta w(p)=\sum_{i=1}^{n} \Delta t_{i}(p)$. Note that if $\delta\left(\mu_{0}, w\right)=\mu_{1}, w \in S e q\left(\mu_{0}\right)$, then $\mu_{1}=\mu_{0}+\Delta w$ as a vector. 


\section{Some codes related to Petri nets}

For a Petri net $P N=(P, A, W, \mu)$ and a subset $X \subseteq \operatorname{Re}(\mu)$ we can define a deterministic automaton $A(P N)$ as follows: $\operatorname{Re}(\mu), A, \delta: \operatorname{Re}(\mu) \times A \rightarrow \operatorname{Re}(\mu)$, $\mu$, and $X$, are regarded as the state set, the input set, the next-state function, the initial state, and the final set of $A(P N)$, respectively (For basic concepts of automata, refer to [1,p.10]). By using such automata, in [9] we defined four kinds of prefix codes and examined fundamental properties of these codes.

The set

$$
\operatorname{Stab}(P N)=\{w \mid w \in \operatorname{Seq}(\mu) \text { and } \delta(\mu, w)=\mu\}
$$

forms a submonoid of $A^{*}$. If $\operatorname{Stab}(P N) \neq\{1\}$, then we denote the base of $\operatorname{Stab}(P N)$ by $S(P N)$. Since $S(P N) A^{+} \cap S(P N)=\emptyset, S(P N)$ is a prefix code over A.

A submonoid $M$ of $A^{*}$ is called pure [6] if for all $x \in A^{*}$ and $n \geq 1$,

$$
x^{n} \in M \Longrightarrow x \in M \text {. }
$$

A subsemigroup $H$ of a semigroup $S$ is extractable in $S$ [8, p.191] if

$$
x, y \in S, z \in H, x z y \in H \Longrightarrow x y \in H .
$$

Proposition 2.1. $\operatorname{Stab}(P N)$ is an extractable pure monoid.

Proof. It is clear that $\operatorname{Stab}(P N)$ is right unitary. Let $y, x y \in \operatorname{Stab}(P N)$. Then $x$ is a sequence from the initial marking $\mu$. Since $\Delta y=0$ (the zero vector) and $\Delta(x y)=\Delta x+\Delta y=0$, we have $x \in \operatorname{Stab}(P N)$. Thus $\operatorname{Stab}(P N)$ is left unitary. Therefore $\operatorname{Stab}(P N)$ is biunitary.

Assume that $x^{n} \in \operatorname{Stab}(P N), n \geq 1$. Then it is obvious that $x$ is a squence from $\mu$. Since $\Delta\left(x^{n}\right) \Delta x=0$, we have $\Delta x=0$ DThus $x \in \operatorname{Stab}(P N)$, and $\operatorname{Stab}(P N)$ is pure.

Let $x, y \in A^{*}$ and $z, x z y \in \operatorname{Stab}(P N)$. If $x=1$, then $z, z y \in \operatorname{Stab}(P N)$. Since $\operatorname{Stab}(P N)$ is biunitary, we have $y \in \operatorname{Stab}(P N)$ and $x y \in \operatorname{Stab}(P N)$. Similarly $y=1$ implies $x y \in \operatorname{Stab}(P N)$. Suppose that $x, y \in A^{+} . y$ is a sequence from $\mu+\Delta x z=\mu+\Delta x$. Thus $x y$ is a squence from $\mu$. From $\mu+\Delta(x z y)=\mu+\Delta(x y)=\mu$ we have $x y \in \operatorname{Stab}(P N)$.

Definition 2.1. Let $P N=(P, A, W, \mu)$ be a Petri net with a positive marking $\mu$. Define the subset $D(P N)$ as the set of all positive sequence $w$ of $S(P N)$.

Since $D(P N)$ is a subset of a bifix code $S(P N)$, also $D(P N)$ is a bifix code over $A$ if $D(P N) \neq \emptyset$. By the same argument mentioned above, we have

Proposition 2.2. If $D(P N) \neq \emptyset$, then $D(P N)^{*}$ is an extractable pure monoid.

Example 2.1. Let $P N=(\{p, q\},\{a, b\}, W, \mu)$ be a Petri net defined by $W(a, p)=W(p, b)=W(q, a)=W(b, q)=1, \mu(p)=\mu(q)=2$. Then $D(P N)=$ 
$\{a b, b a\}$, therefore $\{a b, b a\}^{*}$ is pure $[1$, p.324].

Proposition 2.3. If $x, y \in A^{+}, z, x z y \in D(P N)$, then $x z^{*} y \subseteq D(P N)$.

Proof Let $x, y \in A^{+}, z, x z y \in D(P N)$ and $\mu$ an initial marking of $P N$.

First we show that $x y \in D(P N)$. $x$ is a positive sequence from $\mu$, and $y$ is a positive sequence from $\mu+\Delta(x z)=\mu+\Delta x$. Therefore $x y \in P S e q(\mu)$. Since $\Delta(x z y)=\Delta(x y)=0$, we have that $x y \in D(P N)^{*}$, so that $x y=d_{1} \cdots d_{m}$ for some $d_{i} \in D(P N), m \geq 1$. We have the following three cases.

Case $(a) . m=1, x y=d_{1} \in D(P N)$

Case (b). $m \geq 2, x=d_{1} u$ for some $u \in A^{*}$.

Case (c). $m \geq 2, d_{1}=x u, y=u v, v=d_{2} \cdots d_{m}$ for some $u, v \in A^{*}$.

In Case (b) we have $d_{1}, x z y=d_{1} u z y \in D(P N)$, but it dose not occur since $D(P N)$ is a prefix code. In Case $(c)$, if $u=1$, then $d_{1}, x z y=d_{1} z y \in D(P N)$ DThis contradicts the fact that $D(P N)$ is a prefix code. Therefore $u \neq 1$ DIt follows that both $d_{m}$ and $x z y=x z u d_{2} \cdots d_{m}$ are elements of $D(P N)$. This contradicts the fact that $D(P N)$ is a suffix code. Therefore only Case $(a)$ is possible to occur. Thus $x y \in D(P N)$.

Next we show that $x, y \in A^{+}, z, x z y \in D(P N)$ implies $x z^{2} y \in D(P N)$. Since $z$ is a positive sequence from $\mu+\Delta x=\mu+\Delta(x z)$, we have $x z^{2} \in P \operatorname{Seq}(\mu)$. Since $y$ is a positive sequence from $\mu+\Delta(x z)=\mu+\Delta\left(x z^{2}\right)$, we have $x z^{2} y \in P \operatorname{Seq}(\mu)$. Therefore, from $\Delta\left(x z^{2} y\right)=\Delta(x z y)=0$ we have $x z^{2} y \in D(P N)^{*}$ DThus

$$
x z^{2} y=d_{1} \cdots d_{m}, d_{i} \in D(P N), m \geq 1 .
$$

We have the following four cases.

Case 1. $m=1, x z^{2} y=d_{1} \in D(P N)$,

Case 2. $m \geq 2, d_{1}=x z^{2} u, y=u d_{2} \cdots d_{m}$ for some $u \in A^{*}$,

Case 3. $m \geq 2, d_{1}=x z u, z=u v, v y=d_{2} \cdots d_{m}$ for some $u, v \in A^{*}$,

Case 4. $m \geq 2, d_{1}=x u, z=u v, v z y=d_{2} \cdots d_{m}, u, v \in A^{*}$ for some $u, v \in A^{*}$,

Case 5. $m \geq 2, x=d_{1} u$, for some $u \in A^{*}$.

In Case $2, d_{m}, x z y=x z u d_{2} \cdots d_{m} \in D(P N)$. Thus Case 2 cannot occur since $D(P N)$ is a suffix code. In Case $3, d_{m} \in D(P N)$, and $x z y=x u v y=x u d_{2} \cdots d_{m} \in$ $D(P N)$. However Case 3 cannot occur since $D(P N)$ is a suffix code. Since $D(P N)$ is a prefix code, Case 4 and Case 5 cannot occur. Therefore only Case 1 is possible to occur. Thus $x z^{2} y \in D(P N)$.

Now suppose that $x, y \in A^{+}, z, x z^{n} y \in D(P N), n \geq 2$. Then, $x z^{n-1}, y \in A^{+}$, $z,\left(x z^{n-1}\right) z y \in D(P N)$. Therefore we have $\left(x z^{n-1}\right) z^{2} y=x z^{n+1} y \in D(P N)$.

Let $C$ be a code over $A$. $C$ is an infix code ([7,p.129]), if for all $x, y, z \in A^{*}$,

$$
z, x z y \in C \Longrightarrow x=y=1,
$$

Proposition 2.4. If $D(P N)$ is a non-empty finite set, then $D(P N)$ is an infix code. 
Proof Let $x, y \in A^{*}, z, x z y \in D(P N) . x=1, y \neq 1$ or $x \neq 1, y=1$ cannot ocuur because $D(P N)$ is a bifix code. Therefore either $x=y=1$ or $x, y \in A^{+}$. By Proposition 2.3, $x, y \in A^{+}$and $z, x z y \in D(P N)$ follow that $x z^{*} y \in D(P N)$. This contradicts the fact that $D(P N)$ is a finite set. Thus we have $x=y=1$.

Example 2.2. (1). Let $P N=\left(\{p, q, r\},\{a, b, c, d\}, W, \mu_{0}\right)$ be a Petri net such that $W(p, a)=W(a, q)=W(q, b)=W(b, r)=W(r, c)=W(c, q)=W(q, d)=$ $W(d, p)=1, \quad \mu_{0}=(2,1,1)$. Then $D(P N)=a(b c)^{*} d$. Therefore the infinite code $D(P N)$ is infix. Thus the converse of Proposition 2.4 is false.

$(2)$. Let $P N=\left(\{p\},\{a, b\}, W, \mu_{0}\right)$ be a Petri net such that $W(a, p)=1, W(p, b)=$ $1, \mu_{0}=(1)$. Then $a b, a^{2} b^{2} \in D(P N)$. Therefore the infinite code $D(P N)$ is not infix.

\section{Limited code}

A submonoid $M$ of $A^{*}$ is very pure if for all $u, v \in A^{*}$,

$$
u v, v u \in M \Rightarrow u, v \in M \text {. }
$$

The base of a very pure monoid is called a circular code.

Let $p, q \geq 0$ be two integers. A code $C$ is called $(p, q)$-limited if for any sequence $u_{0}, u_{1}, \ldots, u_{p+q}$ of words in $A^{*}$, the assumptions $u_{i-1} u_{i} \in C^{*}(1 \leq i \leq p+q)$ imply $u_{p} \in C^{*}$.

Any limited code is circular ([1, p.329, Proposition 2.1]). If a subset $C$ of $A^{*}$ is a bifix (1,1)-limited code, then for any $u_{0}, u_{1}, u_{2} \in A^{*}$ such that $u_{0} u_{1}, u_{1} u_{2} \in C^{*}$ we have $u_{1} \in C^{*}$. Thus $u_{0} u_{1}, u_{1}, u_{1} u_{2} \in C^{*}$. This imples that $u_{0}, u_{1}, u_{2} \in C^{*}$ because $C^{*}$ is biunitary. Therefore $C$ is $(p, q)$-limited for all $p, q$ with $p+q=2$.

Let $P N_{0}=\left(\{p\},\{a, b\}, W, \mu_{0}\right)$ be a Petri net such that $W(a, p)=\alpha, W(p, b)=$ $\beta, \mu_{0}=\left(\lambda_{p}\right), \lambda_{p}>0$.

Consider the set $\Omega$ of all positive markings in $P N_{0}$;

$$
\Omega=\left\{\mu \mid \mu=\mu_{0}+\Delta w, w \in P \operatorname{Seq}\left(\mu_{0}\right\} .\right.
$$

Let $g=\operatorname{gcd}(\alpha, \beta)$ be the greatest common divisor of $\alpha$ and $\beta$, and let $\mathbb{N}=$ $\{0,1,2, \cdots\}$ be the set of non-negative integers. Then we have

(0) $D\left(P N_{0}\right)$ is dense, that is, $D\left(P N_{0}\right) \cap A^{*} w A^{*} \neq \emptyset$ for every $w \in A^{*}$.

(1) If $\lambda_{p}<g$, then $\Omega=\left\{\lambda_{p}+n g \mid n \in \mathbb{N}\right\}$.

(2) If $\lambda_{p}=s g, s \geq 1, s \in \mathbb{N}$, then $\Omega=\{n g \mid n \geq 1, n \in \mathbb{N}\}$.

(3) If $\lambda_{p}=s g+t_{p}, s \geq 0,0<t_{p}<g$, then $\Omega=\left\{t_{p}+n g \mid n \geq 0, n \in \mathbb{N}\right\}$. 
Proposition 3.1. If $\lambda_{p}>\operatorname{gcd}(\alpha, \beta)$, then $D\left(P N_{0}\right)$ is not circular.

Proof. Let $D=D\left(P N_{0}\right)$, and let $g=\operatorname{gcd}(\alpha, \beta)$. Note that $\mu_{0}=\lambda_{p}$. We have the following two cases:

Case 1. $g=\alpha$ or $g=\beta$. Case 2. $\alpha=\alpha^{\prime} g, \beta=\beta^{\prime} g, \alpha^{\prime} \geq 2, \beta^{\prime} \geq 2, \operatorname{gcd}\left(\alpha^{\prime}, \beta^{\prime}\right)=1$.

Case 1-(i). If $\alpha=\operatorname{gcd}(\alpha, \beta), \beta=k \alpha, k>1$, then $a a^{k-1} b, a^{k-1} b a \in D$ and $a \notin D^{*}$. Therefore $D$ is not circular.

Case 1-(ii). If $\beta=\operatorname{gcd}(\alpha, \beta), \alpha=k \beta, k>1$, then $a b^{k-1} b, b a b^{k-1} \in D$ and $b \notin D^{*}$. Thus $D$ is not circular.

Case 1-(iii). If $\alpha=\operatorname{gcd}(\alpha, \beta), \alpha=\beta$, then $a b, b a \in D$. Consequently $D$ is not circular.

Case 2. Since $g=\operatorname{gcd}(\alpha, \beta)$, there exist some integers $x^{\prime}$ and $y^{\prime}$ such that $\alpha x^{\prime}+\beta y^{\prime}=g$.

Case 2-(i). We consider the case $\alpha x^{\prime}+\beta y^{\prime}=g, x^{\prime}>0, y^{\prime}<0$. We set $x=x^{\prime}, y=$ $-y^{\prime}$, then $\alpha x-\beta y=g, x>0, y>0$. Since $\alpha x=\beta y+g>\beta i$, for $i=1, \cdots, y, b^{y}$ is a sequence from $\lambda_{p}+\Delta\left(a^{x}\right)$, and $\lambda_{p}+\Delta\left(a^{x} b^{y}\right)=\lambda_{p}+g$. Consequently $a^{x} b^{y}$ is also a sequence from $\lambda_{p}+\Delta\left(a^{x} b^{y}\right)$, therefore $\left(a^{x} b^{y}\right)^{2} \in P S e q\left(\mu_{0}\right)$. Similarly we have $\left(a^{x} b^{y}\right)^{\beta^{\prime}} \in P \operatorname{Seq}\left(\mu_{0}\right)$ and $\lambda_{p}+\Delta\left(\left(a^{x} b^{y}\right)^{\beta^{\prime}}\right)=\lambda_{p}+\beta^{\prime} g$. Thus $\left(a^{x} b^{y}\right)^{\beta^{\prime}} b \in D\left(P N_{0}\right)$. On the other hand, since $\lambda_{p}>g$, we have $\lambda_{p}+\Delta\left(\left(a^{x} b^{y}\right)^{\beta^{\prime}-1} b\right)=\lambda_{p}-g$. It follows that $\left(a^{x} b^{y}\right)^{\beta^{\prime}-1} b \cdot a^{x} b^{y} \in D$. However $a^{x} b^{y} \notin D^{*}$. Thus $D$ is not circular.

Case 2-(ii). We consider the case $-\alpha x+\beta y=g$ for some positive integers $x$ and $y$. Then $a\left(a^{x} b^{y}\right)^{j} \in P \operatorname{Seq}\left(\mu_{0}\right), 1 \leq j \leq \alpha^{\prime}$. Thus $a\left(a^{x} b^{y}\right)^{\alpha^{\prime}} \in D$. On the other hand, from $\lambda_{p}>g$ and $\alpha^{\prime} \geq 2$ we have $\lambda_{p}+\Delta\left(a^{x} b^{y}\right)=\lambda_{p}-g>0$. Thus $a^{x} b^{y} a \in P \operatorname{Seq}\left(\mu_{0}\right)$. It follows that $a^{x} b^{y} a\left(a^{x} b^{y}\right)^{\alpha^{\prime}-1} \in D$. However $a^{x} b^{y} \notin D^{*}$. Therefore $D$ is not circular.

Proposition 3.2. If $\lambda_{p} \leq \operatorname{gcd}(\alpha, \beta)$, then any nonempty subset of $D\left(P N_{0}\right)$ is $(p, q)$-limited for all $p, q$ with $p+q=2$.

Proof. Let $D=D\left(P N_{0}\right)$ and $g=\operatorname{gcd}(\alpha, \beta)$. Let $d$ be an arbitrary element in $D$. Then $d$ has a proper right factor $v \neq 1, d$, because $a, b \notin D$. Let $d=u v$, $u, v \in A^{+} \mathrm{D}$

First, we shall show that $\Delta v \leq-g$. Assume the contrary. Then $\Delta v>-g$, and we have $\Delta v \geq 0$ since $\Delta v$ is a multiple of $g$. If $\Delta v=0$, then $\Delta u=0$ since $\Delta d=\Delta(u v)=\Delta u+\Delta v=0$. Therefore we get $u \in D^{*}$. This contradicts the fact that $D$ is a prefix code. Thus we have $\Delta v>0$, it follows that $\Delta v \geq g$ and $\Delta u=-\Delta(v) \leq-g$. Then we have $\mu_{0}+\Delta u=\lambda_{p}+\Delta u \leq \lambda_{p}-g \leq 0$, showing that $u \notin P \operatorname{Seq}\left(\mu_{0}\right)$ and contradicting $d \in D$. Therefore we have prove $\Delta v \leq-g$.

Next we shall show that any nonempty subset $C$ of $D$ is $(1,1)$-limited. Note that $C$ is a bifix code. Suppose that $u_{0}, u_{1}, u_{2} \in A^{*}$ and $u_{0} u_{1}, u_{1} u_{2} \in C^{*}$. If $u_{i}=1$ for some $i, 0 \leq i \leq 2$, then $u_{0}, u_{1}, u_{2} \in D^{*}$ since $C^{*}$ is biunitary. We assume that $u_{i} \neq 1$ for all $i, 0 \leq i \leq 2$. We may write

$$
u_{0}=v_{0} x_{1}, u_{1}=y_{1} w_{1}, x_{1} y_{1} \in C, v_{0}, w_{1} \in C^{*} .
$$


If $y_{1} \neq 1$ and $y_{1} \notin C$, then $y_{1}$ is a proper right factor of $d_{i} \in D$. Therefore $\Delta\left(y_{1}\right) \leq-g$ as mentioned above. It follows $\lambda_{p}+\Delta y_{1} \leq 0$, and $y_{1} \notin \operatorname{PSeq}\left(\mu_{0}\right)$. However, $u_{1} u_{2}=y_{1} w_{1} u_{2} \in C^{*} \subseteq D^{*}$. Thus $y_{1} \in P \operatorname{Peq}\left(\mu_{0}\right)$. This is a contradiction. Therefore $y_{1}=1$ or $y_{1} \in C$. Thus $u_{1} \in C^{*}$.

Let $P N_{1}=\left(\{p, q\},\{a, b\}, W, \mu_{0}\right)$ be a Petri net such that $W(a, p)=\alpha>$ $0, W(p, b)=\alpha^{\prime}>0, W(q, a)=\beta>0, W(b, q)=\beta^{\prime}>0, \mu_{0}(p)=\lambda_{p}, \mu_{0}(q)=\lambda_{q}$. We examine the code $D\left(P N_{1}\right)$ associated with Petri net $P N_{1}$.

Suppose that $D\left(P N_{1}\right) \neq \emptyset$ and $w \in D\left(P N_{1}\right)$. Let $n=|w|_{a}$ and $m=|w|_{b}$, then $\Delta(w) \Delta(a)+m \Delta(b)=0$. Consequently the linear equation

$$
\left(\begin{array}{cc}
\alpha & -\alpha^{\prime} \\
-\beta & \beta^{\prime}
\end{array}\right)\left(\begin{array}{l}
n \\
m
\end{array}\right)=\left(\begin{array}{l}
0 \\
0
\end{array}\right)
$$

has a non-trivial solution. Thus $\alpha \beta^{\prime}=\alpha^{\prime} \beta$. Therefore, if $D\left(P N_{1}\right) \neq \emptyset$, then $P N_{1}=\left(\{p, q\},\{a, b\}, W, \mu_{0}\right)$ has the following form:

$W(a, p)=\alpha, W(p, b)=k \alpha, W(q, a)=\beta, W(b, q)=k \beta$, for some $k>0$.

Here we assume that $k$ is a positive integer. That is, we define a Petri net $P N_{1}=\left(\{p, q\},\{a, b\}, W, \mu_{0}\right)$ as follows:

$$
\Delta(a)=\left(\begin{array}{r}
\alpha \\
-\beta
\end{array}\right), \quad \Delta(b)=\left(\begin{array}{r}
-k \alpha \\
k \beta
\end{array}\right)
$$

where $k$ is a positive integer.

We define an integer $M_{p}$ as follows

$$
M_{p}= \begin{cases}\frac{\lambda_{p}}{\alpha}-1, & \text { if } \frac{\lambda_{p}}{\alpha} \text { is an integer } \\ {\left[\frac{\lambda_{p}}{\alpha}\right],} & \text { if } \frac{\lambda_{p}}{\alpha} \text { is not an integer }\end{cases}
$$

where [ ] is the symbol of Gauss. Similarly we difine an integer $M_{q}$ as follows, $M_{q}=\frac{\lambda_{q}}{\beta}-1$ if $\frac{\lambda_{q}}{\beta}$ is an integer, and $M_{q}=\left[\frac{\lambda_{p}}{\beta}\right]$ if $\frac{\lambda_{q}}{\beta}$ is not an integer. Note that $a, a^{2}, \cdots, a^{M_{q}} \in P \operatorname{Seq}\left(\mu_{0}\right)$ and $a^{M_{q}+1} \notin P \operatorname{Seq}\left(\mu_{0}\right)$. If $M_{p} \geq k$, then $b \in \operatorname{PSeq}\left(\mu_{0}\right)$. Now, we observe that $M_{p}+M_{q} \leq k-1$ implies $D\left(P N_{1}\right)=\emptyset$. If $M_{p}+M_{q} \leq k-1$, then $M_{p} \leq k-1$. It follows that $b \notin P S e q\left(\mu_{0}\right)$. Furthermore, if $M_{q}=0$, then $a \notin \operatorname{PSeq}\left(\mu_{0}\right)$ and $\operatorname{PSeq}\left(\mu_{0}\right)=\{1\}$. If $M_{q}>0$, we have

$$
a^{i} \in P \operatorname{Seq}\left(\mu_{0}\right), 1 \leq i \leq M_{q} \text {, and } \Delta a^{i}(p)=\lambda_{p}+\alpha i \leq \lambda_{p}+\alpha M_{q} .
$$

Assume that $\lambda_{p}+\alpha M_{q}-\alpha k>0$. If $\frac{\lambda_{p}}{\alpha}$ is an integer, then $M_{p}+1+M_{q}-k>0$. This contradicts the hypothesis. If $\lambda_{p}=\alpha M_{p}+s$ for some $s, 1 \leq s<\alpha$, then

$$
0<M_{p}+M_{q}+\frac{s}{\alpha}-k<M_{p}+M_{q}+1-k
$$


This also contradicts our hypothesis. Therefore we get $\lambda_{p}+\alpha M_{q}-\alpha k \leq 0$, showing that $b$ is not enabled in $\mu_{0}+\Delta\left(a^{i}\right), 1 \leq i \leq M_{q}$. Therefore $\operatorname{PSeq}\left(\mu_{0}\right)=$ $\left\{1, a, a^{2}, \cdots, a^{M_{q}}\right\}$. Thus the condition $M_{p}+M_{q} \leq k-1$ implies $D\left(P N_{1}\right)=\emptyset$.

Lemma 3.3. Let $d=u v \in D\left(P N_{1}\right), u, v \in A^{+}$.

(1) If $M_{p}=0$ and $M_{q} \geq k$, then $\Delta v(p) \leq-\alpha$.

(2) If $M_{p} \geq k$ and $M_{q}=0$, then $\Delta v(q) \leq-\beta$.

Proof. (1). Suppose that $\Delta v(p)>-\alpha$. Then, since $\Delta v(p)$ is a multiple of $\alpha$, we have $\Delta v(p) \geq 0$. Note that

$$
\Delta v=|v|_{a} \Delta a+|v|_{b} \Delta b=\left(\begin{array}{r}
\left(|v|_{a}-k|v|_{b}\right) \alpha \\
-\left(|v|_{a}-k|v|_{b}\right) \beta
\end{array}\right) .
$$

If $\Delta v(p)=0$, then $|v|_{a}-k|v|_{b}=0$. Thus $\Delta v=0$, it follows that $\Delta u=0$ and $u \in D\left(P N_{1}\right)^{*}$. This contradicts the fact that $D\left(P N_{1}\right)$ is a prefix code. Thus $\Delta v(p) \geq \alpha$. It implies that $\Delta u(p)=-\Delta v(p) \leq-\alpha$. Since $M_{p}=0$, $\mu_{0}(p)+\Delta u(p)<\lambda_{p}-\alpha \leq 0$. This yields $u \notin P S e q\left(\mu_{0}\right)$. This is a contradiction. Therefore we have $\Delta v(p) \leq-\alpha$.

(2). Proof is omitted.

Proposition 3.4. We have

(1) If $M_{p}+M_{q}>k, M_{p} \geq k$ and $M_{q} \geq 1$, then $D\left(P N_{1}\right)$ is not circular.

(2) If $M_{p}+M_{q}>k, k>M_{p} \geq 1, M_{q}>1$, then $D\left(P N_{1}\right)$ is not circular.

(3) If $M_{p}+M_{q}=k$, then $D\left(P N_{1}\right)$ is a singleton.

(4) If $M_{p}=0, M_{q}>k$, then any nonempty subset of $D\left(P N_{1}\right)$ is $(p, q)$-limited for all $p, q$ with $p+q=2$.

(5) If $M_{p}>k, M_{q}=0$, then any nonempty subset of $D\left(P N_{1}\right)$ is $(p, q)$-limited for all $p, q$ with $p+q=2$.

Proof. Let $D=D\left(P N_{1}\right)$.

(1) From $M_{p} \geq k$ it follows that $b \in P S e q\left(\mu_{0}\right)$ and $b a^{k} \in D$. On the other hand, from $\lambda_{p}>k \alpha$ we have $\lambda_{p}+\alpha-k \alpha>\alpha$. Thus $a b \in P \operatorname{Seq}\left(\mu_{0}\right)$. Since $\lambda_{p}+(k-1) \beta>(k-1) \beta$, we have $a b a^{k-1} \in D$. Therefore $D$ is not circular.

(2) Let $k=M_{p}+r$ DSince $M_{p}+M_{q}>k$, we have $M_{q}>r$. It follows that $a^{r} \in P \operatorname{Seq}\left(\mu_{0}\right)$. Since $\lambda_{p}+r \alpha-k \alpha=\lambda_{p}-\alpha M_{p}>0$, we have $a^{r} b \in \operatorname{PSeq}\left(\mu_{0}\right)$. Consequently $a^{r} b a^{M_{p}} \in D$. On the other hand we have $a^{r+1} b a^{M_{p}-1} \in D$ since $M_{q}>r$. Therefore $D$ is not circular.

(3) First we consider the case that $M_{p} \geq 1, M_{q} \geq 1$. Since $M_{p}=k-M_{q}<k$, we have $b \notin P \operatorname{Seq}\left(\mu_{0}\right)$. It is obvious that $a^{i} \in P \operatorname{Seq}\left(\mu_{0}\right)$ for $i=0,1, \cdots, M_{q}$. If $i \leq M_{q}-1$, then from $\lambda_{p}+i \alpha-k \alpha=\lambda_{p}-M_{p} \alpha-\left(M_{q}-i\right) \alpha$ and $\lambda_{p}-M_{p} \alpha \leq \alpha$, we get $\lambda_{p}+i \alpha-k \alpha \leq 0$, showing that $a^{i} b \notin P S e q\left(\mu_{0}\right), 0 \leq i \leq M_{q}-1$. It is obvious that $a^{M_{q}} b \in P \operatorname{Seq}\left(\mu_{0}\right)$ and

$$
\mu_{0}+\Delta\left(a^{M_{q}} b\right)=\left(\begin{array}{c}
\lambda_{p}-\alpha M_{p} \\
\lambda_{q}+\beta M_{p}
\end{array}\right)
$$


For $j=0, \cdots, k-M_{q}$, we have $a^{M_{q}} b a^{j} \in P S e q\left(\mu_{0}\right)$ since $\lambda_{q}+\beta M_{p}-\beta j \geq \lambda_{q}$. However $a^{M_{q}} b a^{j} b \notin P \operatorname{Seq}\left(\mu_{0}\right)$ since

$$
\lambda_{p}-\alpha M_{p}+\alpha j \leq \lambda_{p}-\alpha M_{p}+\alpha\left(k-M_{q}\right)=\lambda_{p} \leq k \alpha .
$$

Therefore $D=\left\{a^{M_{q}} b a^{k-M_{q}}\right\}$. Similarly, if $M_{p}=0, M_{q}=k$, then $D=\left\{a^{k} b\right\}$. If $M_{p}=k, M_{q}=0$, then $D=\left\{b a^{k}\right\}$.

(4) Let $C$ be a nonempty subset of $D$. Suppose that $w_{0}, w_{1}, w_{2} \in A^{*}$ and $w_{0} w_{1}, w_{1} w_{2} \in C^{*}$. If $w_{i}=1$ for some $i, 0 \leq i \leq 2$, then $w_{0}, w_{1}, w_{2} \in C^{*}$ since $C^{*}$ is biunitary. We assume that $w_{i} \neq 1$ for all $i, 0 \leq i \leq 2$. We may write

$$
w_{0}=u_{0} x_{1}, w_{1}=y_{1} v_{1}, x_{1} y_{1} \in C \text { for some } x_{1}, y_{1} \in A^{*}, u_{0}, v_{1} \in C^{*} .
$$

If either $y_{1}=1$ or $y_{1} \in C$, then $w_{1} \in C^{*}$. Assume that $y_{1} \neq 1$ and $y_{1} \notin C$. Then $y_{1}$ is a proper right factor of an element in $D$. Since $M_{p} \leq \alpha, \lambda_{p}+\Delta y_{1} \leq 0$ by Lemma 3.3, we have $y_{1} \notin P \operatorname{Seq}\left(\mu_{0}\right)$. However $w_{1} w_{2}=y_{1} v_{1} w_{2} \in C^{*} \subset D^{*}$. Thus $y_{1} \in \operatorname{PSeq}\left(\mu_{0}\right)$. This is a contradiction. Therefore $y_{1} \in C \cup\{1\}$. This yields $w_{1} \in C^{*}$.

(5) The proof of (5) is similar to the proof of (4), therefore it is omitted.

Remark 3.1. In the above proof for (3) we have $D=\{w\}, w=a^{M_{q}} b a^{k-M_{q}}$, $M_{q} \neq 0, k-M_{q} \neq 0$. $D$ is (s, t)-limited for all $s, t \geq 0$ with $s+t=3$. For any $n, m \geq 0$ the code $D=\left\{a^{n} b a^{k-n}\right\}=\left\{a^{n} b a^{m}\right\}, k+m$, is realizable as a Petri net code which is produced by the Petri net $P N_{1}$ such that $W(a, p)=W(q, a)=$ $1, W(p, b)=W(b, q)=k, \mu_{0}(p)=m+1, \mu_{0}(q)+1$.

Let $P N=\left(P, A, W, \mu_{0}\right)$ be a Petri net. By $P R e\left(\mu_{0}\right)$ we denote the set of all possible positive markings reachable from $\mu_{0}$. For a Petri net $P N$ we define a deterministic automaton $A(P N)$ as follows:

$P \operatorname{Re}\left(\mu_{0}\right), A, \delta: P \operatorname{Re}\left(\mu_{0}\right) \times A \rightarrow P R e\left(\mu_{0}\right), \mu_{0}$, and $\left\{\mu_{0}\right\}$, are regarded as the state set, the input set, the next-state function, the initial state, and the final set of $A(P N)$, respectively.

Corollary 3.5. Let $n$ and $k$ be arbitrary integers such that $n>k>1$. Define the automaton

$$
\mathcal{A}_{(n, k)}=(\{1,2, \cdots, n\},\{a, b\}, f, 1,\{1\})
$$

by $f(i, a)=i+1,1 \leq i \leq n-1, f(j, b)=j-k, k+1 \leq j \leq n$. Then any nonempty subset of the base of language $L\left(\mathcal{A}_{(n, k)}\right)$ recognized by $\mathcal{A}_{(n, k)}$ is a $(\mathrm{p}, \mathrm{q})$-limited code for all $p, q$ with $p+q=2$.

Proof. We define the $P N_{1}=\left(\{p, q\},\{a, b\}, W, \mu_{0}\right)$ as follows: $W(a, p)=1, W(p, b)=k, W(b, q)=k, W(q, a)=1, \mu_{0}(p)=1, \mu_{0}(q)$. Then $M_{p}=0, M_{q}-1 \geq k$. Therefore, by Proposition 3.4, $D(P N)$ is $(1,1)$-limited. Since $A\left(P N_{1}\right)$ is isomorphic to $\mathcal{A}_{(n, k)}$ as an automaton, we have Corollary 3.5. 
Proposition 3.6. Let $P N=\left(\left\{p_{1}, \cdots, p_{n}\right\},\left\{a_{1}, \cdots, a_{n}\right\}, W, \mu_{0}\right), n \geq 2$, be a Petri net such that $W\left(p_{i}, a_{i}\right)=\alpha_{i}, W\left(a_{i}, p_{i+1}\right)=\beta_{i}, 1 \leq i \leq n-1$, and $W\left(p_{n}, a_{n}\right)=\alpha_{n}, W\left(a_{n}, p_{1}\right)=\beta_{n}, \quad \mu_{0}=\left(\lambda_{1}, \cdots, \lambda_{n}\right), \mu_{0}\left(p_{i}\right)=\lambda_{i}, 1 \leq i \leq n$. Furthermore let $g_{j}=\operatorname{gcd}\left(\beta_{j-1}, \alpha_{j}\right), 2 \leq j \leq n$. If $\lambda_{1} / \alpha_{1}>1$ and $\lambda_{i} \leq g_{i}$ for all $i=2, \cdots, n$, and if $D(P N) \neq \emptyset$, then any nonempty subset of $D(P N)$ is $(p, q)$ limited for all $p, q$ with $p+q=2$.

Proof. We set $D=D(P N)$. Since $\lambda_{i} \leq g_{i}$ for all $i=2, \cdots, n$, we have $D \subset a_{1} A^{+}$. Let $d \in D, d=a u v, u \in A^{*}, v \in A^{+}$DNote that $v$ is a proper right factor of an element in $D$.

First we show that $\Delta v\left(p_{i}\right) \leq 0$ for all $i=2, \cdots, n$. Suppose that $\Delta v\left(p_{j}\right)>0$ for some $j \geq 2$. Since $\Delta v\left(p_{j}\right)>0$ is a linear combination of $\beta_{j-1}$ and $\alpha_{j}, \Delta v\left(p_{j}\right)$ is a multiple of $g_{j}$. Therefore $\Delta v\left(p_{j}\right)>0$ implies $\Delta(v)\left(p_{j}\right) \geq g_{j}$. Thus $-\Delta v\left(p_{j}\right) \leq-g_{j}$. On the other hand, $\Delta d=\Delta\left(a_{1} u\right)+\Delta v=0$, and we have $\Delta\left(a_{1} u\right)=-\Delta v$. Therefore $\Delta\left(a_{1} u\right)\left(p_{j}\right)=-\Delta v\left(p_{j}\right) \leq-g_{j}$. However, $\mu_{0}\left(p_{j}\right)+\Delta\left(a_{1} u\right)\left(p_{j}\right) \leq \lambda_{j}-g_{j} \leq 0$. This contradicts the fact that $a_{1} u \in P \operatorname{Seq}\left(\mu_{0}\right)$. Consequently we have that $\Delta v\left(p_{i}\right) \leq 0$ for all $i,(i \geq 2)$.

Next we show that $v \notin P S e q\left(\mu_{0}\right)$. To prove this we show that there exists some $p_{t}, t \geq 2$, such that $\Delta v\left(p_{t}\right) \leq-g_{t}$. Suppose the contrary. Then $\Delta v\left(p_{i}\right)=0$ for all $i \geq 2$. Let $x_{j}$ be the number of the letter $a_{j}$ in $v$, then

$$
\Delta(v)=\left(\begin{array}{ccccc}
-\alpha_{1} & 0 & \cdots & 0 & \beta_{n} \\
\beta_{1} & -\alpha_{2} & \cdots & 0 & 0 \\
0 & \beta_{2} & \cdots & 0 & 0 \\
& & \cdots & & \\
0 & 0 & \cdots & \beta_{n-1} & -\alpha_{n}
\end{array}\right)\left(\begin{array}{c}
x_{1} \\
x_{2} \\
\cdot \\
\cdot \\
x_{n}
\end{array}\right)=\left(\begin{array}{c}
\Delta(v)\left(p_{1}\right) \\
0 \\
\cdot \\
\cdot \\
0
\end{array}\right)
$$

We regard the equation above as a system of linear equations. Since $D \neq \emptyset$, the determinant of a matrix $\left(\Delta a_{1}, \cdots, \Delta a_{n}\right)$ is zero. That is, $\alpha_{1} \alpha_{2} \cdots \alpha_{n}=\beta_{1} \beta_{2} \cdots \beta_{n}$. Since there exists a solution, we must have $\Delta v\left(p_{1}\right)=0$. Consequently $\Delta\left(a_{1} u\right)=$ $-\Delta v=0$ and $a_{1} u \in P \operatorname{Seq}\left(\mu_{0}\right)$. Therefore $a_{1} u \in D^{*}$. This contradicts the fact that $D$ is a prefix code. Thus we have proved that $\Delta v\left(p_{t}\right) \leq-g_{t}$ for some $p_{t}, t \geq 2$. This means that $v \notin P S e q\left(\mu_{0}\right)$ since $\mu_{0}\left(p_{t}\right)+\Delta(v)\left(p_{t}\right) \leq \lambda_{j}-g_{t} \leq 0$.

Finally we prove that any nonempty subset $C$ of $D$ is $(1,1)$-limited. Suppose that $w_{0}, w_{1}, w_{2} \in A^{*}$, and $w_{0} w_{1}, w_{1} w_{2} \in C^{*}$. We may write

$$
w_{0}=u_{0} x_{1}, w_{1}=y_{1} v_{1}, x_{1} y_{1} \in C \text { for some } x_{1}, y_{1} \in A^{*}, u_{0}, v_{1} \in C^{*}
$$

Note that $y_{1}$ is a right factor of an element of $D$. If $y_{1} \neq 1$ and $y_{1} \notin C$, then $y_{1} \notin P \operatorname{Seq}\left(\mu_{0}\right)$ as we mentioned above. Therefore $w_{1} w_{2}=y_{1} v_{1} w_{2} \notin \operatorname{PSeq}\left(\mu_{0}\right)$. This contradicts our hypothesis. Thus $y_{1}=1$ or $y_{1} \in C$. This shows that $w_{1} \in C^{*}$. 
Let $P N_{2}=\left(\left\{p_{1}, p_{2}\right\},\{a, b, c\}, W, \mu_{0}\right)$ be a Petri net such that $W\left(a, p_{1}\right)=\alpha_{1}, W\left(p_{1}, b\right)=\alpha_{2}, W\left(b, p_{2}\right)=\beta_{1}, W\left(p_{1}, c\right)=\alpha_{3}, W\left(p_{2}, c\right)=\beta_{2}$, $\mu_{0}\left(p_{1}\right)=\lambda_{1}, \mu_{0}\left(p_{2}\right)=\lambda_{2}$.

Lemma 3.7. Let $P N_{2}$ be a Petri net mentioned above, and let $\alpha=\operatorname{gcd}\left(\alpha_{1}, \alpha_{2}, \alpha_{3}\right), \beta=\operatorname{gcd}\left(\beta_{1}, \beta_{2}\right)$. Suppose that $D\left(P N_{2}\right) \neq \emptyset$ and $\lambda_{1} \leq \alpha, \lambda_{2} \leq$ $\beta$. If $d \in D\left(P N_{2}\right)$ and $v, v \neq 1$, is a proper right factor of $d$, then we have one of the following:

(1) $\Delta v\left(p_{1}\right) \leq-\alpha, \Delta v\left(p_{2}\right) \leq-\beta$.

(2) $\Delta v\left(p_{1}\right)=0, \Delta v\left(p_{2}\right) \leq-\beta$.

(3) $\Delta v\left(p_{1}\right) \leq-\alpha, \Delta v\left(p_{2}\right)=0$.

Proof. Let $D=D\left(P N_{2}\right)$. It is obvious that $b$ and $c$ are not enabled in $\mu_{0}$, so that $D \subset a A^{*}$. Let $d \in D, d=a u v, u \in A^{*}, v \in A^{+}$. If $u=1$, then $\Delta v=-\Delta a$ and (3) holds. Assume $u \neq 1$. If $\Delta v\left(p_{1}\right)>0$, then $\Delta v\left(p_{1}\right) \geq \alpha$ because $\Delta v\left(p_{1}\right)$ is a multiple of $\alpha$. Thus $\Delta(a u)\left(p_{1}\right)=-\Delta v\left(p_{1}\right) \leq-\alpha$. Hence $\mu_{0}\left(p_{1}\right)+\Delta(a u)\left(p_{1}\right) \leq \mu_{0}\left(p_{1}\right)-\alpha \leq 0$. This contradicts the fact that $a u \in P \operatorname{Seq}\left(\mu_{0}\right)$. Therefore $\Delta v\left(p_{1}\right) \leq 0$. Similarly we have $\Delta v\left(p_{2}\right) \leq 0$. If $\Delta v\left(p_{1}\right)=0$ and $\Delta v\left(p_{2}\right)=0$, i.e., $\Delta v=0$, then $\Delta(a u)=0$. Since $a u \in P S e q\left(\mu_{0}\right)$, we have $a u \in D^{*}$, contradicting the fact that $D$ is a prefix code. Therefore at least one of $(1),(2)$ or (3) occurs.

Proposition 3.8. If $D\left(P N_{2}\right) \neq \emptyset$ and $\lambda_{1} \leq \alpha, \lambda_{2} \leq \beta$, then any nonempty subset of $D\left(P N_{2}\right)$ is $(p, q)$-limited for all $p, q$ with $p+q=2$.

Proof. Let $D=D\left(P N_{2}\right)$. We note that for a right factor $v, v \neq 1$, of an element $d \in D$ the vector $\mu_{0}+\Delta v$ is not positive by Lemma 3.7. That is, $v \notin P \operatorname{Seq}\left(\mu_{0}\right)$. Let $C$ be a nonempty subset of $D$. Assume $w_{0}, w_{1}, w_{2} \in A^{*}$ and $w_{0} w_{1}, w_{1} w_{2} \in C^{*}$. If either $w_{0}=1$ or $w_{1}=1$, then $w_{1} \in C^{*}$. Therefore we consider the case where $w_{0} \neq 1$ and $w_{1} \neq 1$. Let $w_{0} w_{1}=d_{1} \cdots d_{m}, d_{i} \in C, 1 \leq i \leq m$. There exist an integer $i, 1 \leq i \leq m$, and $u, v \in A^{*}$ such that $w_{0}=d_{1} \cdots d_{i-1} u, d_{i}=u v$, $w_{1}=v d_{i+1} \cdots d_{m}$. If $v \neq 1$ and $v \notin D$, then $v$ is a proper right factor $d_{i}$. Thus $v \notin P \operatorname{Seq}\left(\mu_{0}\right)$. However, from $w_{1} w_{2}=v d_{i+1} \cdots d_{m} w_{2} \in C^{*}$, we heve $v \in \operatorname{PSeq}\left(\mu_{0}\right)$. This is a contradiction. Hence $v=1$ or $d \in C$. It follows that $w_{1} \in C^{*}$. Thus $C$ is (1,1)-limited.

Let $P N_{3}=\left(\{p, q\},\{a, b, c\}, W, \mu_{0}\right)$ be a Petri net such that $W(a, p)=\alpha$, $W(q, a)=\beta, W(p, b)=\alpha+\beta, W(b, q)=\alpha+\beta, W(c, p)=\beta, W(q, c)=\alpha, \mu_{0}(p)=$ $\lambda_{p}, \mu_{0}(q)=\lambda_{q}$.

Lemma 3.9. Let $P N_{3}$ be a Petri net mentioned above. If $\beta<\lambda_{p} \leq \alpha+\beta$ and $\beta<\lambda_{q} \leq \alpha$, then for any $u \in \operatorname{PSeq}\left(\mu_{0}\right)$ we have one of the following:

(1) $\Delta u=\left(\begin{array}{c}k(\alpha-\beta) \\ k(\alpha-\beta)\end{array}\right), k \geq 0, \quad(2) \Delta u=\left(\begin{array}{c}k(\alpha-\beta)+l \alpha \\ k(\alpha-\beta)-l \beta\end{array}\right), k \geq 0, l \geq 1$ 
(3) $\Delta u=\left(\begin{array}{c}k(\alpha-\beta)-l \beta \\ k(\alpha-\beta)+l \alpha\end{array}\right), k \geq 0, l \geq 1$.

Proof. We shall prove the lemma by induction on the length of $u$ in $P S e q\left(\mu_{0}\right)$. Since $\beta<\lambda_{p} \leq \alpha+\beta$, and $\beta<\lambda_{q} \leq \alpha$, only $a$ is enabled in $\mu_{0}$. That is, a positive sequence of length 1 is only $a$, and $\Delta a$ is of the form (2).

Since $\lambda_{q}-\beta<\alpha-\beta<\alpha, c$ is not enabled in $\mu_{0}+\Delta a . \Delta\left(a^{2}\right)$ is of the form (2), and $\Delta(a b)=(-\beta, \alpha)$ is of the form (3). $c$ is not enabled in $\mu_{0}+\Delta\left(a^{2}\right) . b$ is not enabled in $\mu_{0}+\Delta(a b) . \Delta\left(a^{3}\right)$ is of form (2). $\Delta\left(a^{2} b\right), \Delta(a b a)$ and $\Delta(a b c)$ are of the form (1).

Now we suppose that for $u \in P \operatorname{Seq}\left(\mu_{0}\right),|u|>3$, the vector $\Delta u$ has a form $(1),(2)$ or (3). Let $x$ be an element in $\{a, b, c\}$ such that $u x \in P S e q\left(\mu_{0}\right)$. We shall show that $\Delta(u x)$ is of the form (1),(2) or(3).

Case 1. $\Delta u=(k(\alpha-\beta), k(\alpha-\beta)) . \Delta(u a)$ is of the form (2). If $k=0$, then both $b$ and $C$ are not enabled in $\mu_{0}+\Delta u$. For $k \geq 1, \Delta(u b)=((k-1)(\alpha-\beta)-2 \beta,(k-$ $1)(\alpha-\beta)+2 \alpha)$ is of the form (3). $\Delta(u c)=((k-1)(\alpha-\beta)+\alpha,(k-1)(\alpha-\beta)-\beta)$ is of the form (2).

Case 2. $\Delta u=(k(\alpha-\beta)+l \alpha, k(\alpha-\beta)-l \beta) . \Delta(u a)$ is of the form (2). If $l=1$, then $\Delta(u b)$ is of the form (3). If $l \geq 2$, then $\Delta(u b)=((k+1)(\alpha-\beta)+(l-$ $2) \alpha,(k+1)(\alpha-\beta)-(l-2) \beta)$ is the form (1) or (2). If $k=0$, then $c$ is not enabled in $\mu_{0}+\Delta u$. For $k \geq 1, \Delta(u c)=((k-1)(\alpha-\beta)+2 \alpha,(k-1)(\alpha-\beta)-2 \beta)$.

Case 3. $\Delta u=(k(\alpha-\beta)-l \beta, k(\alpha-\beta)+l \alpha) . \Delta(u a)=((k+1)(\alpha-\beta)-(l-$ 1) $\beta,(k+1)(\alpha-\beta)+(l-1) \alpha)$ is of the form (1) or (3). $\Delta(u c)=(k(\alpha-\beta)-(l-$ $1) \beta, k(\alpha-\beta)+(l-1) \alpha)$. If $k=0$, then $b$ is not enabled in $\mu_{0}+\Delta u$. For $k \geq 1$, $\Delta(u b)=((k-1)(\alpha-\beta)-(l+2) \beta,(k-1)(\alpha-\beta)+(l+2) \alpha)$. Thus Lemma 3.9 is proved.

Proposition 3.10. If $D\left(P N_{3}\right) \neq \emptyset$, and if $\beta<\lambda_{p} \leq \alpha+\beta$ and $\beta<\lambda_{q} \leq \alpha$, then any nonempty subset of $D\left(P N_{3}\right)$ is $(p, q)$-limited for all $p, q$ with $p+q=2$.

Proof. Let $D=D\left(P N_{3}\right)$, and let $v, v \neq 1$, be a proper right factor of $d \in D$.

First we shall show that $v \notin P S e q\left(\mu_{0}\right)$. Let $d=u v, u, v \in A^{+}$, then $\Delta v=-\Delta u$. Since $u \in P \operatorname{Seq}\left(\mu_{0}\right)$, by Lemma 3.9 we have one of the following:

(i) $\Delta v=\left(\begin{array}{c}-k(\alpha-\beta) \\ -k(\alpha-\beta)\end{array}\right), k \geq 0, \quad$ (ii) $\Delta v=\left(\begin{array}{c}-k(\alpha-\beta)-l \alpha \\ -k(\alpha-\beta)+l \beta\end{array}\right), k \geq 0, l \geq 1$,

(iii) $\Delta v=\left(\begin{array}{c}-k(\alpha-\beta)+l \beta \\ -k(\alpha-\beta)-l \alpha\end{array}\right), k \geq 0, l \geq 1$.

We consider Case (iii). If $v \in P S e q\left(\mu_{0}\right)$, then, by Lemma 3.9 we have the following three cases. Case (iii)-(1)

$$
\Delta v=\left(\begin{array}{c}
-k(\alpha-\beta)+l \beta \\
-k(\alpha-\beta)-l \alpha
\end{array}\right)=\left(\begin{array}{c}
x(\alpha-\beta) \\
x(\alpha-\beta)
\end{array}\right), k \geq 0, l \geq 1, x \geq 0 .
$$


In this case, there is not a solution for $x \mathrm{D}$ Case (iii)-(2)

$$
\Delta v=\left(\begin{array}{c}
-k(\alpha-\beta)+l \beta \\
-k(\alpha-\beta)-l \alpha
\end{array}\right)=\left(\begin{array}{c}
x(\alpha-\beta)+y \alpha \\
x(\alpha-\beta)-y \beta
\end{array}\right), k \geq 0, l \geq 1, x \geq 0, y \geq 1 .
$$

In this case, only one solution of linear system is a non-positive $(x, y)=(-(k+l), l)$. Case (iii)-(3)

$$
\Delta v=\left(\begin{array}{c}
-k(\alpha-\beta)+l \beta \\
-k(\alpha-\beta)-l \alpha
\end{array}\right)=\left(\begin{array}{c}
x(\alpha-\beta)-y \beta \\
x(\alpha-\beta)+y \alpha
\end{array}\right), k \geq 0, l \geq 1, x \geq 0, y \geq 1 .
$$

In this case, only one solution of linear system is a non-positive $(x, y)=(-k,-l)$. Therefore in any cases we have $v \notin P \operatorname{Seq}\left(\mu_{0}\right)$. Similarly, in Case (i) or Case (ii) we cannot write $\Delta v$ in the form (1), (2) or (3) of Lemma 3.9. Therefore $v \notin P S e q\left(\mu_{0}\right)$.

Let $C$ be a subset of $D$. Assume $w_{0}, w_{1}, w_{2} \in A^{*}$ and $w_{0} w_{1}, w_{1} w_{2} \in C^{*}$. If either $w_{0}=1$ or $w_{1}=1$, then $w_{1} \in C^{*}$. Therefore we consider the case where $w_{0} \neq 1$ and $w_{1} \neq 1$. Let $w_{0} w_{1}=d_{1} \cdots d_{m}, d_{j} \in C, 1 \leq j \leq m$. There exist an integer $i, 1 \leq i \leq m$, and $u, v \in A^{*}$ such that $w_{0}=d_{1} \cdots d_{i-1} u, d_{i}=u v$, $w_{1}=v d_{i+1} \cdots d_{m}$. If $v \neq 1$ and $v \neq d_{i}$, then $v$ is a proper right factor $d_{i}$. By using the above fact that $v \notin P \operatorname{Seq}\left(\mu_{0}\right)$, we obtain $w_{1} \notin P \operatorname{Seq}\left(\mu_{0}\right)$. This is a contradiction. Thus we have either $v=1$ or $v=d_{i}$ which implies $w_{1} \in C^{*}$. Thus $C$ is (1,1)-limited.

When a submonoid of a free monoid is given, it seems complicated to judge whether the submonoid is pure or not. This is because we have to show it by the treatment of many different cases of words which belong to the submonoid. Also it doesn't seem easy to decide whether the base of a pure monoid is limited or not. Proposition 2.1 and 2.2 ensure that any submonoid generated by a code $D(P N)$ or $S(P N)$ is always pure. The proof techniques of Proposition 3.2-3.10 which use the properties of right factors of the elements in $D(P N)$ may be usable to decide whether $D(P N)$ is limited or not in other Petri nets.

\section{References}

[1] Berstel, J., and Perrin, D. Theory of Codes. Academic Press, 1985.

[2] Ito, M., and Kunimochi,Y. Some Petri Net Languages and Codes. Lecture Note in Computer Science, 2295:69-80, 2002.

[3] Lallement, G. Semigroup and Combinatorial Applications. Wiley, 1979.

[4] Murata, T. Petri Nets: Properties, Analysis and Applications. Proceeding of the IEEE, 77(4):541-580, 1989.

[5] Peterson, J. L. Petri Net Theory and the Modeling of Systems. Prentice Hall, 1981. 
[6] Restivo, A. On a Question of McNaughton and Papert. Information and Control, 25:93-101, 1974.

[7] Shyr, H. J. Free monoids and languages. Hon Min Book Company, Taichung, Taiwan, 1991.

[8] Tamura, T. Hangunron, Kyoritu-Shuppan, Tokyo, 1972. (In Japanese)

[9] Tanaka, G. Prefix codes determined by Petri nets. Algebra Colloquium, $5(3): 255-264,1998$.

[10] Tanaka, G. Circular codes and Petri nets. In Shoji, K. (ed.) Proc. 9th Symposium on Algebras, Languages and Computation, RIMS Kokyuroku, 1503:132$138,2006$.

Received 8th May 2008 